\title{
Dynamics of Domain Walls in Magnetic Semi-conductors with Applied Magnetic Fields and Currents
}

1. H. Ohno, "A window on the future of spintronics. Nature Materials 9952 (2010)

2. Jae-Chui Lee et al., "Role of adiabatic and nonadiabatic spin transfer torqes on magnetic domain wall motion. http://arxiv.org/abs/1006.1216

\section{Recommendation and Commentary by Thierry Giamarchi, University of Geneva}

In many systems the domain walls separating two different regions of the order parameter play a central role. This is obviously the case in magnetic or ferroelectric systems, but also occur in others such as magnetic semiconductors. Although domain walls can be viewed as a nuisance, they are in fact crucial in the practical use of these materials since by manipulating them one determines how information can be stored. Understanding the dynamics of such domain walls is thus crucial. Given the fact that there is disorder in the materials leading to pinning of the domain walls this is a beautiful fundamental physics problem as well.

Taking into account the full microscopic details would be totally untractable and thus simplified models focussing on the physics of the wall itself were developed. The most naive model is to consider the domain wall as a string (for simplicity let us consider a $1 \mathrm{D}$ wall in a film, i.e. a $2 \mathrm{D}$ material) whose position can be determined by a displacement field $u(z)$, a function of the height in the direction parallel to the wall. Since having a domain wall costs energy there is an elastic energy associated to the domain wall, often modeled as $\int d z \frac{c}{2}\left(\nabla_{z} u(z)\right)^{2}$ and a pinning energy taking into a account the fact that the wall moves in a random potential $V(x, z)$ symbolizing the microscopic disorder in the system. This pinning energy is simply $\int d z V(u(z), z)$. Such a competition between elastic energy, which would like the wall to be flat, and disorder which wants the wall to meander leads to

glassy properties which are crucial when one looks at the dynamics. The motion of the wall can be described by the equation

$$
\eta \partial_{t} u(z)=-\frac{\partial H}{\partial u(z)}+F_{\text {ext }}+\zeta(z, t)
$$

This equation describes overdamped motion with a friction $\eta$ coming from 
internal dissipation mechanism inside the domain wall and with a force $F_{\text {ext }}$ setting the wall into motion. For a magnetic domain wall this can be produced by e.g. putting the system in a magnetic field which favors one of the regions of the order parameter (or an electric field for a ferroelectric). $\zeta(z, t)$ is a thermal (Langevin) noise which is supposed to mimic the effects of the temperature. It is important that this noise has fluctuation that are precisely connected to the dissipation $\left.\eta \overline{\left(\zeta(x, t) \zeta\left(x^{\prime}, t^{\prime}\right)\right.}=\eta T \delta\left(x-x^{\prime}\right) \delta\left(t-t^{\prime}\right)\right)$ in order to satisfy the fluctuation dissipation theorem.

Solving this equation is of course a formidable problem. However when the applied force is small, a remarkable behavior occurs which can be obtained by either phenomenological arguments or a renormalization group solution of the above equation. The velocity $v$ of the driven domain wall is given by:

$$
v \propto e^{-\beta U_{c}\left(F_{c} / F_{\text {ext }}\right)^{\mu}}
$$

where $U_{c}$ and $F_{c}$ are characteristic quantities, and $\mu$ a universal exponent. This remarkable non-linear response at small drive, called creep, has its roots in the glassy properties of the domain wall, and comes from the fact that the system has to overcome divergent barriers by Arhhenius activation in order to be able to move. Quite remarkably this behavior has been experimentally verified over many orders of magnitude in magnetic and ferroelectric domain walls [1].

One could thus be lulled in believing that we understand everything in this problem. Fortunately this is far from being the case. The approximation which consists in modeling the domain walls by a simple position is clearly an oversimplification for several materials. For magnetic systems, old works by Walker [2] have already pointed that in addition to the domain wall one had to retain the angle $\phi$ that the spin makes perpendicular to the direction of the order parameter. One has thus to write a second equation similar to (1) for the angle $\phi$. This already poses some non-trivial conceptual problems about where to put the dissipation and how to write the thermal noise. A priori one could expect two dissipation constants $\eta_{1}$ and $\eta_{2}$, and there is already fierce debate in the community on what is the underlying mechanism behind this dissipation and what the values can be.

This is specially important for systems such as the magnetic semiconductors, currently intensely studied in connection with spintronics. Indeed for these materials it is possible to transfer (e.g. by spin-orbit coupling) the moment coming from the spin of moving electrons to the domain wall. It is thus possible to have a magnetic domain wall move either by driving it by a magnetic field $B$ (the situation we were discussing up to now) or by 
passing an electrical current $J$. This second driving force would appear in the second equation for the angle $\phi$.

One immediate question is of course what happens now of the law of Eq.(2) depending on whether the external force is the magnetic field or the current. Does one have creep for the two type of forces, or another form of response? Are the exponents identical? Given the complexity of the problem on the theoretical side it is difficult to go beyond simple phenomenological derivations [3]. On the experimental side conflicting results were obtained $[4,5]$, but seemed to point out towards a different behavior depending on the driving force, magnetic field or current. More recent studies seem to be in agreement with the differing behavior of the two driving forces (Ref.1 above), although no details were given. The recent paper (Ref.2 above) on the contrary seem to point out towards the same universal behavior for the two drives and in agreement with the creep theory. The nature of the difference of behavior between the two studies is at that stage unclear. Whether this is due to a difference in the materials, and hence in the intrinsic mechanisms for spin relaxation, remains to be elucidated.

In all cases these papers point out the difficulty in understanding the dynamics in situation where several variables are important in the equation

of motion. This can occur in spintronic materials because of the coupling between the position of the wall $u$ and the spin angle $\phi$ but is also likely to occur in materials such as multiferroics [6] for which the domain wall is made of two different types of order (ferroelectic and magnetic). The study of such dynamical situations with coupling between several degrees of freedom is thus bound to provide many headaches and hopefully beautiful results for the future.

\section{References}

[1] P. Metaxas et al., Phys. Rev. Lett. 99, 217208 (2007), and refs. therein.

[2] N. L. Schryer and L. R. Walker, J. Applied Phys. 45, 5406 (1974).

[3] R. A. Duine and C. Morais Smith, Phys. Rev. B 77, 094434 (2008)

[4] M. Yamanouchi et al., Science 3171726 (2007).

[5] J. P. Adam et al., Phys. Rev. B 80, 193204 (2009).

[6] H. Bea and P. Paruch, Nature Materials 8168 (2009). 